\title{
Psychometric Properties of the Medication Non-Adherence Questionnaire in Patients With Psychiatric Disorders
}

\author{
Masoud Bahreini ${ }^{1}$; Zahra Rafiee ${ }^{2,}$; Shahnaz Pouladi ${ }^{1}$; Kamran Mirzaei ${ }^{3}$; Masoud \\ Mohammadi Baghmollaei ${ }^{4}$ \\ ${ }^{1}$ Department of Nursing, Bushehr University of Medical Sciences, Bushehr, IR Iran \\ ${ }_{2}^{2}$ Student Research Committee, Department of Nursing, Bushehr University of Medical Sciences, Bushehr, IR Iran \\ ${ }^{3}$ Department of Social Medicine, Bushehr University of Medical Sciences, Bushehr, IR Iran \\ ${ }^{4}$ Health School, Bushehr University of Medical Sciences, Bushehr, IR Iran \\ ${ }^{*}$ Corresponding author: Zahra Rafiee, Student Research Committee, Department of Nursing, Bushehr University of Medical Sciences, Bushehr, IR Iran. Tel: +98-9398373034, Fax: \\ +98-7714550187, E-mail:Zahra_rafiee67@yahoo.com
}

Received: January 17, 2015; Revised: April 16, 2015; Accepted: May 4, 2015

\begin{abstract}
Background: Non-adherence to medication in psychiatric patients and identification of related risk factors has provided serious challenges for care service providers.

Objectives: The current study aims to determine the psychometrics of a questionnaire used to indicate risk factors related to nonadherence to medication in psychiatric patients.

Patients and Methods: Four-hundred patients with psychiatric disorders in Bushehr and Shiraz were enrolled in this cross-sectional study using convenient sampling methods. An initial questionnaire was designed with 23 items. Following the confirmation of content and face validity of the questionnaire, the questionnaire was completed by the participants and 11 experts contributing to the administration. The item impact score, content validity index (CVI), and content validity ratio (CVR) were examined using exploratory factor analysis. In order to calculate the internal and external reliability, the Kuder-Richardson and re-test methods were used.

Results: Factor analysis revealed five factors in the questionnaire. Five of 23 items had low content validity and were eliminated. The CVI and CVR of the questionnaire were 0.89 and 0.85 , respectively. One statement was eliminated owing to a reduced factor load. Internal reliability was $r=0.86$, estimated using the Kuder-Richardson method, and external reliability was $r=0.93$, estimated via a Pearson correlation coefficient. Five factors resulting from the questionnaire had optimal reliability according to the Cronbach's alpha coefficient (0.79). Five factors were extracted, including factors related to disease, patient and environment, attitudes toward treatment and therapist, drug side effects, and previous experience to treatment.

Conclusions: The questionnaire on risk factors related to medication non-adherence in patients with psychiatric disorders had acceptable psychometric characteristics, and is a useful tool to be implemented in medical centers and psychological clinics.
\end{abstract}

Keywords: Psychometrics; Risk Factors; Medication Adherence; Questionnaires

\section{Background}

Medication non-adherence in psychiatric patients is one of the serious challenges for psychiatrists and health care providers (1). Estimates indicate that $25 \%$ to $80 \%$ of patients with psychiatric disorders are unable to properly use drugs prescribed by physicians (2). It is noted that non-adherence to medication increases the probability of recurrence of symptoms by 3 to 5 times, compared with adherence to medication (3). Non-adherence to medication is responsible for 125,000 deaths in the United States annually (4).

Poor adherence to medication regimens not only leads to a lack of treatment efficacy, but also increases the cost of health care. Non-adherence to a medication regimen can have devastating effects on the patient and his or her family in terms of personal suffering, hospitalization, and reduced quality of life. Non-adherence to medication regimens can also have detrimental effects on society by imposing exorbitant costs of health care (5).

Gaining knowledge about factors related to medication non-adherence helps physicians, nurses, and health care providers design programs for better care, suitable and timely hospital discharge, sustainable treatment, as well as reduced recurrence of disease and need for hospitalization (6). Thus, evaluating medication adherence in patients seems necessary to ensure effective treatment and understand the obstacles for treatment

Copyright (C) 2015, Ahvaz Jundishapur University of Medical Sciences. This is an open-access article distributed under the terms of the Creative Commons Attribution-NonCommercial 4.0 International License (http://creativecommons.org/licenses/by-nc/4.0/) which permits copy and redistribute the material just in noncommercial usages, provided the original work is properly cited. 
efficacy. Evaluation of medication adherence requires applications and reliable tools based on the needs of the target group. If there is access to such tool, it is possible to use it with providing evidence for its reliability, and in case on lack of access to needed tool, it should be designed.

Evaluating medication non-adherence in patients seems necessary to determine the prognosis of treatment and to understand the obstacles in treatment efficacy. Generally, non-adherence measurement methods include self-report, counting the number of used drugs, and biological measurements (7). The Medication Adherence Questionnaire, Beliefs about Medicines Questionnaire, and Medication Adherence Rating Scale are among the commonly used tools for non-adherence measurement $(8,9)$. However, no comprehensive tool that includes all the risk factors related to medication non-adherence has been designed (10).

Mostly common questionnaires have often been used in limited number of studies in this field is the study by Ghaffari-nejad et al. (11) which is conducted to determine factors related to non-adherence in patients with mood disorders. The authors used the DAI questionnaire (11), which evaluates the attitude of patients and does not include other involved factors. Moreover, using a 14-item checklist, Omranifard et al. (9) attempted to identify medication non-adherence factors that lead to re-hospitalization in patients with psychiatric disorders. In this study, the supportive role of the family was not mentioned, and the reliability and validity of the checklist were not evaluated.

Although the supportive role of the family in non-adherence has been referred to in some studies aiming at identifying risk factors related to non-adherence $(1,6)$, this factor has not been duly considered in the common tools. The supportive role of a patient's family or relatives has been neglected in these instruments.

Some studies have been conducted to determine and identify related factors. Mitchell and Selmes (12) conducted a review aiming at investigating factors predicting medication non-adherence in patients with psychiatric disorders. They reported factors related to patients, clinical factors (e.g. physician), and disease, including risk factors in medication non-adherence (12).

Mahtani et al. (13) identified several factors related to medication non-adherence in patients with psychiatric disorders, which included demographic characteristics, relationship between patient and care provider, family support, previous adherence to medication, history of drug abuse, and side effects of drugs (13).

Among few studies addressing the supportive role of family, the study by Abu Rahma et al. entitled "Medication Adherence and Family Supportive Role in Prevention of Recurrence in Patients with Schizophrenia in Gaza" (14) can be mentioned. In their study, patients responded to questionnaires related to the family's supportive role, but it lacked the other section related to determination of this factor to be answered by patients' families or relatives.

Considering the increased prevalence of mental disorders and extent of their clinical consequences, prevention from risk factors and continuation of research on medication non-adherence in psychiatric patients seems necessary. Moreover, the inadequate attention of authors in the domestic area and the limitations of studies on this topic show the importance of addressing this topic. Thus, given the importance of the subject and the lack of a suitable instrument for determination of risk factors related to medication non-adherence in patients with psychiatric disorders, it is necessary to design a questionnaire that has acceptable reliability and validity levels and is compatible with the Iranian culture. As for designing an authentic questionnaire, there is some need for a larger samples size. However, owing to time limitations and inaccessibility of more samples, only samples from Shiraz and Bushehr were used.

\section{Objectives}

The current study aims to design a psychometric questionnaire for determining risk factors related to medication non-adherence in patients with psychiatric disorders.

\section{Patients and Methods}

This study is a cross-sectional inquiry. The research population included all outpatients referring to psychiatric centers and patients discharged from psychiatric wards in Bushehr and Shiraz during 2013, and one of the patients' family members. Inclusion criteria included: the patients' consent and also the consent of the person who accompanied them to the study and complete the questionnaire; minimum age of 15 years; suffering from only one disorder of three disorders including schizophrenia, depression, and bipolar disorder (confirmed by a psychiatrist); history of psychiatric drugs for at least the previous 6 months; and having a companion at the time of reference. Exclusion criteria included: lack of consciousness so that the patient was not able to answer questions; usage of drugs other than psychiatric drugs; and having other diseases. The written consent of the participants was collected.

Various ratios for the necessary sample size for factor analysis have been stated in different studies. A minimum ratio of participants to variables has been reported as 3:1, 10:1, 15:1, or 20:1 in different studies $(15,16)$. Considering the number of items for exploratory factor analysis (18 items), the sample size was specified as 360 participants, which was consistent with the maximum ratio of subject to variable (20:1). Four hundred samples were considered for the study, taking into account the sample loss probability. The sufficient sample size for factor analysis 
was greater than 0.6, according to the Kaiser-Meyer-Olkin measure of sampling adequacy. To ensure adequacy of the sample size of the study, the Kaiser-Meyer-Olkin test and Bartlett tests were performed (17).

\subsection{Questionnaire Design Steps}

The questionnaire was designed in three steps: extraction of questions, determination of the validity, and determination of the reliability.

\subsubsection{Step 1}

In this step, items were designed after reviewing the related papers and collecting questionnaires related to medication non-adherence. The initial questionnaire included 23 items, mostly "yes-no" questions and some open-ended questions.

\subsubsection{Step 2}

After preparing the questions, face, content, and construct validities were assessed. For face validity, the questions were given to 11 experts in psychology, psychiatry and psychiatric nursing. The questions were evaluated in three aspects: level of difficulty, suitability, and ambiguity. Further, 15 people in the target group expressed their ideas regarding fluency and comprehensibility of the questions. To eliminate the invalid items, the item impact score was determined in the next step. If the impact score for each item was above 1.5 , the item was considered suitable and it was preserved (18).

Next, in order to determine content validity, questions were given to 11 psychiatry and psychology professionals. Experts were asked to examine the items in terms of grammar accuracy, correct word usage, and correct placement of the items in the questionnaire. Next, the content validity was examined quantitatively using the content validity ratio (CVR) and content validity index (CVI). The CVI was developed by Lawshe; the necessity of an item in the tool is examined by a three-point Likert Scale. Next, using the following formula and Lawshe table for determining the minimum content value ratio, items with ratios above 0.59 were kept (Equation 1).

$$
\mathrm{CVR}=\frac{\text { Number of necessary answers in each item }-\left(\frac{\text { Total number of participants }}{2}\right)}{\frac{\text { Total number of participants }}{2}}
$$

Furthermore, to determine the CVI, the questions were given to the 11 experts. The questions were worded in such a way that a 4-point Likert scale for relevance, clarity, and simplicity could be used for each item. Next, the CVI was calculated with the following formula (Equation 2).

$$
\text { CVI }=\frac{\text { Number of experts which scored } 3 \text { or } 4 \text { to item }}{\text { Total number of experts }}
$$

Following the calculation of CVI and CVR, five items were eliminated because they had scores below 0.59, and 18 items were kept so that their construct validity could be measured.

In order to determine construct validity, exploratory factor analysis was used. To this end, the 18-item questionnaire was distributed among 400 patients with psychiatric disorders. It was distributed non-randomly based on characteristics considered by the author. Exploratory factor analysis was done via principal component analysis using a varimax rotation. The results from the exploratory factor analysis included six main outputs.

Obtained results from the first, second, third, and the fifth outputs were provided. Following extraction of factors and statements in each factor, the compatibility of these factors with principal components of medication non-adherence was examined.

\subsubsection{Step 3}

In this step, the reliability of the questions was examined. Questions were examined in terms of internal and external reliability. The Kuder-Richardson test was used to determine internal reliability, and external reliability was measured by the test-retest method and the Pearson correlation coefficient. SPSS 19 software was used for data analysis.

\section{Results}

Results obtained by calculating the Item Impact Index showed high impact scores for all items (above 1.5); thus, all items were considered suitable in the view of the target group and kept for the next steps. To determine content validity, CVI and CVR were calculated. The values for CVI and CVR were 0.89 and 0.85 , respectively. Five statements were eliminated because their scores were too low, based on the Lawshe table (0.59), and the number of items was reduced to 18 . Test-retest results showed high external reliability $(\mathrm{r}=0.93)$. Also, using the Kuder-Richardson formula, the findings showed high internal reli$\operatorname{ability}(\mathrm{r}=0.86)$.

The KMO index of 0.8 suggested an adequate sample size for factor analysis. Moreover, the Bartlett test indicated suitability of factor analysis for identifying a factor model structure at $\mathrm{P}<0.0001$, and suggested discoverable relationships between the variables, which were under analysis.

Other parts of the results of exploratory factor analysis include values related to the initial overlap and extraction overlap. Considering that the initial overlap values before factor extraction are 1 for all items, the minimum value for extraction overlap should be 0.3 (17). If it is larger, extracted factors better represent variables. The results of the exploratory factor analysis indicated that the calculated extraction overlap values for all items were 
between 0.340 and 0.825 ; thus, all items were considered suitable.

The other dimensions of exploratory factor analysis included initial eigenvalues, eigenvalues of extracted factors without rotation, and eigenvalues of extracted factors with rotation (Table 1). In this study, considering eigenvalues larger than 1 and the scree plot slope (Figure 1), five factors with prediction power of $60.12 \%$ were extracted. These factors described changes in risk factors.

Factor analysis also showed a rotated matrix of elements, which included factor loads of each variable in the remaining factors following the rotation (Table 2).

Based on the results obtained from the factor analysis with varimax rotation, five factors were extracted. As shown in Table 2, the questionnaire with five factors was identified. The first factor, with an eigenvalue of 4.652 , included three items, and their factor load varied between 0.859 and 0.900 . The second factor, with an eigenvalue of 1.724 , included seven items, and their factor load varied between 0.374 and 0.745 . The third factor, with an eigenvalue of 1.375 , included three items, and their factor load varied between 0.534 and 0.823 . The fourth factor, with an eigenvalue of 2.337 , included two items, and their factor load varied between 0.759 and 0.840 . The last factor, with an eigenvalue of 1.133 included two items, and their factor load varied between 0.797 and 0.800 . It should be noted that item 4 was assigned to Group 2 considering its content and despite its lower factor load compared to factor 1 . Thus, based on exploratory factor analysis on 18 items, 17 items were confirmed and put into five factors (Table 2 ).

Following factor analysis, the total internal consistency of the questionnaire with 17 items was investigated. Cronbach's alpha coefficient was calculated for the sample size of 400 patients with psychiatric disorders. Chronbach's alpha coefficient was 0.79 for the whole question- naire, with a minimum of 0.53 and maximum of 0.9 for questionnaire factors (Table 3 ).

The questionnaire was developed in two-point Likert scale (Yes/No) format with a score range of 1 to 2, with a of score 1 representing choice Yes and a score of 2 representing choice No. Some questions had inverse scoring. Minimum and maximum scores were 17 and 34. If a score was higher than 17 , more risk factors threatened the patient.

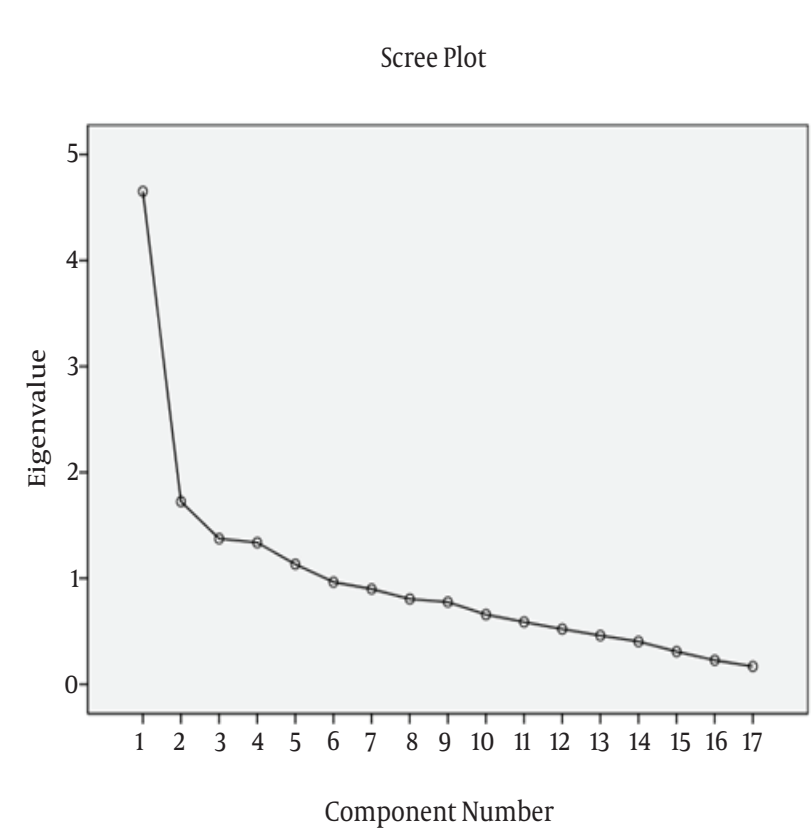

Figure 1. Scree Plot Based on Exploratory Factor Analysis to Determine Correlation Between Items

Table 1. Initial and Extraction Overlap in Exploratory Factor Analysis for Tool in Determining Risk Factors Related to Medication NonAdherence

\begin{tabular}{|c|c|c|c|c|c|c|c|c|c|}
\hline \multirow[t]{2}{*}{ Factor } & \multicolumn{3}{|c|}{ Initial Eigenvalue } & \multicolumn{3}{|c|}{$\begin{array}{c}\text { Eigenvalue of Extraction Factors } \\
\text { Without Rotation }\end{array}$} & \multicolumn{3}{|c|}{$\begin{array}{c}\text { Eigenvalue of Extraction Factors } \\
\text { With Rotation }\end{array}$} \\
\hline & Total & $\begin{array}{l}\text { Variance } \\
\text { Percent }\end{array}$ & $\begin{array}{l}\text { Cumulative } \\
\text { Percentage }\end{array}$ & Total & $\begin{array}{l}\text { Variance } \\
\text { Percent }\end{array}$ & $\begin{array}{l}\text { Cumulative } \\
\text { Percentage }\end{array}$ & Total & $\begin{array}{l}\text { Variance } \\
\text { Percent }\end{array}$ & $\begin{array}{l}\text { Cumulative } \\
\text { Percentage }\end{array}$ \\
\hline 1 & 4.652 & 27.367 & 27.367 & 4.652 & 27.367 & 27.367 & 3.315 & 19.500 & 19.500 \\
\hline 2 & 1.724 & 10.138 & 37.506 & 1.724 & 10.138 & 37.506 & 2.305 & 13.559 & 33.059 \\
\hline 3 & 1.375 & 8.086 & 45.592 & 1.375 & 8.086 & 45.529 & 1.652 & 9.716 & 42.775 \\
\hline 4 & 1.337 & 7.868 & 53.460 & 1.337 & 7.868 & 53.460 & 1.543 & 9.077 & 51.852 \\
\hline 5 & 1.133 & 6.664 & 60.124 & 1.133 & 6.664 & 60.124 & 1.406 & 8.272 & 60.124 \\
\hline
\end{tabular}


Table 2. Rotated Matrix of Elements of Exploratory Factor Analysis for Tool Determining Risk Factors Related to Medication NonAdherence

\begin{tabular}{ccccc}
\hline Items of Tool & Related to & Related to Patient and \\
Disease & Environment & $\begin{array}{c}\text { Attitude to Treatment } \\
\text { and Therapist }\end{array}$ & $\begin{array}{c}\text { Drug Side } \\
\text { Effects }\end{array}$ & $\begin{array}{c}\text { Previous Experience } \\
\text { Toward Treatment }\end{array}$
\end{tabular}

1. In your opinion, do you suffer $\quad 0.900$
from mental disorders?

2. Is your patient aware of his need $\quad 0.864$
for using drugs?

3. Are you aware of disease
symptoms?

4. Are you forced to accompany your $\quad 0.625 \quad 0.410$
patient to drugstore to ensure he prepares prescription?

5. Have you stopped your drugs without physician's order? (arbitrary refusal of drug usage)

\author{
6. Have you had any prescription \\ which you decided not to comply \\ with?
}

7. In case of emergence of annoying complications from drug use, would you continue using it?

8. Are you forced to have direct moni- $\quad 0.510$
toring of drug usage to ensure your
patient takes his medicine?

9. Have you ever decided to use alcohol or other substances instead of taking prescribed medicine?
10. Do you often forget regular use of drugs?

11. Do you believe drug usage helps to reduce or improve symptoms of disease?

\begin{tabular}{l|l|l}
$\begin{array}{l}\text { 12. Do you believe you need medica- } \\
\text { tion to improve your disease? }\end{array}$ & 0.486 \\
$\begin{array}{l}\text { 13. Does the patient tend to refer to } \\
\text { the physician in due time? }\end{array}$ & 0.534 \\
\hline
\end{tabular}
the physician in due time?

14. Has your patient talked about side effects of drugs such as strange movements, sleepiness, weight gain, sexual problems, and blurred vision following using medications?

15. Has your patient talked about side effects of drugs, such as strange movements, sleepiness, weight gain, sexual problems, and blurred vision following the use of medications?

16. Does usage of current drugs by 0.499 
Bahreini M et al.

Table 3. Cronbach's Alpha Coefficient of Questionnaire (Risk Factors Associated With Medication Non-Adherence) After Exploratory Factor Analysis

\begin{tabular}{lcc}
\hline Risk Factors & Number of Statements & Cronbach's Alpha \\
\hline Factors related to disease & 3 & 0.90 \\
\hline Factors related to patient and environment & 7 & 0.71 \\
\hline Attitudes toward treatment and therapist & 3 & 0.56 \\
Drug side effects & 2 & 0.59 \\
\hline Previous experience toward treatment & 2 & 0.53 \\
Whole questionnaire & 17 & 0.79 \\
\hline
\end{tabular}

\section{Discussion}

The results indicated that the designed tool had suitable reliability and validity for determining risk factors related to medication non-adherence in patients with psychiatric disorders. Based on these findings, five factors were extracted from the risk factors questionnaire related to medication non-adherence including factors related to disease, patient and environment, attitude toward treatment and therapist, drug side effects, and previous experience toward treatment. Gabriel et al. (19) referred to four aspects of factors influencing non-adherence, including amnesia, feeling of recovery, feeling of exacerbation, and carelessness. Some of the above-mentioned items are in line with the research in the present study. For example, an item for determining the role of side effects or impact of previous treatments was designed for both questionnaires. However, there were also some inconsistencies: for example, an item entitled amnesia in their questionnaire included factors such as age, while, in the present study, the item related to diseases was designed separately with specific questions. Demographic factors such as age and gender were designed separately at the beginning of the questionnaire considering the local culture of the country, which is an advantage of the present study. In a study by Abu Rahma et al. (14), three tools were designed. These tools are known as the medication adherence scale, family support scale, and recurrence scale (14). In designing questions related to the first tool, the factors were divided into factors related to attitude and adherence. Despite the high number of questions, which is a limitation, they did not include all involved factors. For example, there was no item covering drug abuse. The current questionnaire is more comprehensive, despite its limited number of questions and brief answers (Yes/No). Also, in the study by Abu Rahma et al. (14), the reliability of answers about the supportive role of family is doubtful because the questions related to this part were answered by patients with schizophrenia. One of the advantages of the current research is that the section related to the family was completed by family members.

Unfortunately, there are few studies on medication nonadherence in our country, which have been carried out mostly on non-psychiatric patients (20). Further, most studies address evaluation of non-adherence and do not refer to the design of the tool. Omranifard et al. (9) studied factors related to non-adherence that led to re-hospitalization of 500 patients with psychiatric disorders in Isfahan. Data were collected using checklists including 14 items (items taken from reviewing foreign papers) related to non-adherence. However, the instrument used in the study lacked adequate information regarding its reliability, and no attempt was made to localize the checklist according to the national culture (9).

The reliability results for our tool are higher compared to that in the study by Gabriel et al. (19), which described an antidepressant adherence scale $(r=0.66)$. On the other hand, the reliability coefficient in our study is lower compared to that in the study by Abu Rahma et al. (14) $(r=0.88)$, owing to the type of study (cross-sectional), research population, dissimilarity of tool items, and type of patients under study, considering their disease severity and stage.

The present research study attempted to provide accurate information on the instrument by determining its reliability and validity levels. Useful and brief Yes/No questions and a relatively extensive research population (including three types of diseases) are other strengths of the current research.

The questionnaire was divided into two separate sections (questions related to the patient and the family). The supportive role of the family of the patients with psychiatric disorders was addressed, thus making it applied and useful. The questionnaire on risk factors related to medication non-adherence is the only specialized tool related to psychiatric patients that includes two aspects associated with the patient and patient's relatives, thus making it a point of strength.

Considering that studies on medication non-adherence are mostly performed using samples with one type of disease and with a limited sample size, generalization of findings and applications of the tool in other psychiatric patients is difficult. Therefore, the use of a larger statistical population and variety of samples in terms of the type of disease could have increased tool generalization in this work. 


\subsection{Limitations}

As a limitation, the lack of evaluation of the designed tool in patients with other psychiatric disorders (such as anxiety disorder, personality disorder, etc.) made the generalization of results difficult. Hence, replication of this study is suggested with similar and more extensive samples. Also, further studies on risk factors affecting medication non-adherence and extending the distribution of study population are suggested.

Findings suggest that the designed questionnaire encompasses the optimal psychometric characteristics and possesses the necessary strength and reliability to determine risk factors related to medication non-adherence in patients with psychiatric disorders. The questionnaire can be applied by health care authorities in care and health systems throughout the country. This tool is useful for psychiatrists, psychiatric nurses and psychologists who are employed in dealing with psychiatric patients. This tool can be used by doctors and nurses to examine the many consequences of non-adherence to treatment in patients, which are to be avoided. The questionnaire is compatible to Iranian culture and it is easy and comprehensible, and can be answered by patients with psychiatric disorders and their families.

\section{Acknowledgements}

The current paper results from research findings in a Master's Thesis in Nursing, Bushehr University of Medical Sciences. All professors, psychiatric professionals, as well as patients and their families are highly appreciated. Also, the Clinical Research Development Center of Bushehr Persian Gulf Hospital, which provided useful consulting, is appreciated.

\section{Authors' Contributions}

Study concept and design: Zahra Rafiee, Masoud Bahreini and Shahnaz Pouladi. Analysis and interpretation of data: Shahnaz Pouladi and Kamran Mirzaei. Critical revision: Shanaz Pouladi and Masoud Bahreini. Data gathering: Zahra Rafiee and Masoud Mohammadi Baghmollaei.

\section{Funding/Support}

We appreciate the Deputy of Research of Bushehr University of Medical Sciences, who supported this research financially.

\section{References}

1. Vermeire E, Hearnshaw H, Van Royen P, Denekens J. Patient ad- herence to treatment: three decades of research. A comprehensive review. J Clin Pharm Ther. 2001;26(5):331-42.

2. Priebe S, Yeeles K, Bremner S, Lauber C, Eldridge S, Ashby D, et al. Effectiveness of financial incentives to improve adherence to maintenance treatment with antipsychotics: cluster randomised controlled trial. BMJ. 2013;347:f5847.

3. Staring AB, Mulder CL, Duivenvoorden HJ, De Haan L, Van der Gaag M. Fewer symptoms vs. more side-effects in schizophrenia? Opposing pathways between antipsychotic medication compliance and quality of life. Schizophr Res. 2009;113(1):27-33.

4. Atreja A, Bellam N, Levy SR. Strategies to enhance patient adherence: making it simple. MedGenMed. 2005;7(1):4.

5. Weiden PJ, Olfson M. Cost of relapse in schizophrenia. Schizophr Bull. 1995;21(3):419-29.

6. Stewart SL, Baiden P. An exploratory study of the factors associated with medication nonadherence among youth in adult mental health facilities in Ontario, Canada. Psychiatry Res. 2013;207(3):212-7.

7. Thompson K, Kulkarni J, Sergejew AA. Reliability and validity of a new Medication Adherence Rating Scale (MARS) for the psychoses. Schizophrenia Research. 2000;42(3):241-7.

8. George J, Mackinnon A, Kong DC, Stewart K. Development and validation of the Beliefs and Behaviour Questionnaire (BBQ). Patient Educ Couns. 2006;64(1-3):50-60.

9. Omranifard V, Yazdani M, Yaghoubi M, Namdari M. Noncompliance and its causes resulting in psychiatric readmissions. Iran J of Psychiatry. 2008;3(1):37-42.

10. van Dulmen S, Sluijs E, van Dijk L, de Ridder D, Heerdink R, Bensing J. Patient adherence to medical treatment: a review of reviews. BMC Health Serv Res. 2007;7:55.

11. Ghaffari-Nejad A, Mashayekhi A, Mazhari S, Ghayoumi A. Factors Associated With Drug Non-Adherence After Discharge Among Patients With Mood Disorders Hospitalized in Shahid Beheshti Hospital in Kerman: A Prospective Study. Substance abuse. 2015;57:38.

12. Mitchell AJ, Selmes T. Why don't patients take their medicine? Reasons and solutions in psychiatry. Advances in Psychiatric Treatment. 2007;13(5):336-46.

13. Mahtani KR, Heneghan CJ, Glasziou PP, Perera R, Mahtani KR. Reminder packaging for improving adherence to self-administered long-term medications. Cochrane Database Syst Rev. 2011;9(9).

14. Abu Rahma HM. Drug compliance and family support contribute in preventing relapse among schizophrenia clients in Gaza strip. Islamic university of Gaza Univ. 2014. Available from: http://library. iugaza.edu.ps/thesis/106993.pdf.

15. Westen D, Rosenthal R. Quantifying construct validity: two simple measures.J Pers Soc Psychol. 2003;84(3):608-18.

16. Thompson B. Exploratory and confirmatory factor analysis: Understanding concepts and applications.USA: American Psychological Association; 2004.

17. Pirasteh A, Hidarnia A, Asghari A, Faghihzadeh S, Ghofranipour F. Development and validation of psychosocial determinants measures of physical activity among Iranian adolescent girls. BMC Public Health. 2008;8:150.

18. Lacasse Y, Godbout C, Series F. Health-related quality of life in obstructive sleep apnoea. Eur Respir J. 2002;19(3):499-503.

19. Gabriel A, Violato C. Knowledge of and attitudes towards depression and adherence to treatment: the Antidepressant Adherence Scale (AAS). J Affect Disord. 2010;126(3):388-94.

20. Minaiyan M, Taheri M, Mirmoghtadaee P, Marasi M. Comparative Role of Demographic Factors and Patient's Belief about Prescribed Medicine on Adherence to Drug Treatment in Chronic Diseases. Journal of Isfahan Medical School. 2011;29(156). 